\title{
Obtaining Cubatures for Rectangles and Other Planar Regions by Using Orthogonal Polynomials*
}

\author{
By Richard Franke
}

\begin{abstract}
A. H. Stroud has recently shown the existence of cubature formulas for planar regions which use $m^{2}$ points and have polynomial precision $2 m-1$. In this paper, the author gives sufficient conditions for the existence of formulas using fewer than $\boldsymbol{m}^{2}$ points, and having polynomial precision $2 m-1$. An algorithm is given for computing such formulas, and is shown to be useful in a more general setting than given in the theorem. Numerical examples are given, both in terms of previously known and new cubature formulas.
\end{abstract}

1. Introduction. A number of authors have attempted to use the common zeros of orthogonal polynomials in two variables as evaluation points in cubature formulas for regions in the plane. A theorem given recently by Stroud [10] shows how to construct formulas using orthogonal polynomials of degree $m$ if they have exactly $m^{2}$ distinct common zeros. These formulas approximate the integrals of polynomials of degree $\leqq 2 m-1$ exactly. The principal result of this paper is to give sufficient conditions for the existence of similar formulas which use fewer than $m^{2}$ points.

The following notation and definitions will be used in this paper.

$\boldsymbol{R}_{2} \quad$ A region in the plane.

$w \quad$ A weight function defined, and nonnegative, on $R_{2}$ such that the integrals $\int_{R}, w x^{\alpha} y^{\beta}$ exist for all $\alpha, \beta \geqq 0$.

$P_{d}, P_{d, i} \quad$ Polynomials of degree $d$.

$Q_{d}, Q_{d, i} \quad$ Polynomials of degree $\leqq d$.

$P^{(\alpha, \beta)} \quad$ The orthogonal polynomials of the form $x^{\alpha} y^{\beta}+Q_{m-1}(x, y)$.

$S_{d, 2} \quad$ A subset of pairs of nonnegative integers; $\{(\alpha, \beta): \alpha+\beta \leqq d, \alpha, \beta \geqq 0\}$.

$S_{N} \quad$ A subset of $S_{d, 2}$ containing $N$ elements.

$\left(x_{i}, y_{i}\right) \quad$ A common zero of two polynomials.

$X\left(S_{N}\right) \quad$ The matrix having rows $\left(x_{1}^{\alpha} y_{1}^{\beta}, x_{2}^{\alpha} y_{2}^{\beta}, \cdots, x_{r}^{\alpha} y_{r}^{\beta}\right)$, where $(\alpha, \beta) \in S_{N}$, and If $f) \quad=\int_{R}, w f$.

The cubature formulas we obtain will have the form

$$
I(f) \cong \sum_{k=1}^{N} A_{k} f\left(\mu_{k}\right) .
$$

Received September 4, 1970.

AMS 1970 subject classifications. Primary 41A55, 41A63, 65D30; Secondary 41A10.

Key words and phrases. Approximate integration, cubature formula, orthogonal polynomials, algebraic function, common zeros, positive weights, polynomial precision, rectangles, planar regions.

* This work forms an essential portion of the author's thesis for the Ph.D. degree in Mathematics at the University of Utah, written under the direction of Professor Robert E. Barnhill. The author was supported by a NASA Traineeship for the duration of this research. 
We say that $P_{d}$ is an orthogonal polynomial, with respect to a given region $R_{2}$ and weight function $w$, if $I\left(P_{d} Q_{d-1}\right)=0$ for all $Q_{d-1}$. A cubature formula is said to have precision $d$ if the approximation (1) is exact for all $Q_{d}$ and is not exact for all $P_{d+1}$. A formula is said to be fully symmetric if the appearance of a point $(a, b)$ in the formula implies the appearance of the points $( \pm a, \pm b),( \pm b, \pm a)$ with the same weight. The set of points $\{( \pm a, \pm b),( \pm b, \pm a)\}$ will be denoted by $(a, b)_{\mathrm{FS}}$.

2. Preliminary Discussion. The following theorem was given by Stroud [10].

THEOREM 1. Assume $P_{m, 1}$ and $P_{m, 2}$ are two polynomials in two variables with the following properties:

(i) Each $P_{m, i}$ is orthogonal to all $Q_{m-1}$; and

(ii) $P_{m, 1}$ and $P_{m, 2}$ have exactly $m^{2}$ common zeros, $\nu_{k}, k=1, \cdots, m^{2}$, all of which are distinct and none of which are at infinity.

Then there exist constants $A_{k}$ such that

$$
I\left(Q_{2 m-1}\right)=\sum_{k=1}^{m^{2}} A_{k} Q_{2 m-1}\left(\nu_{k}\right)
$$

for all $Q_{2 m-1}$.

Numerous examples of this theorem can be given, including both previously known and new formulas. The cross product formulas for rectangles are a special case. Some particularly interesting examples are those which effectively use fewer than $m^{2}$ points by virtue of the fact that one or more of the weights are zero. The goal of this investigation is to give conditions ensuring the existence of formulas using fewer than $m^{2}$ points. These formulas do not always occur as a consequence of zero weights appearing in Theorem 1, however.

The weights of the cubature formula (2) can be found by solving the linear system of equations obtained by making (2) exact for some set of $m^{2}$ monomials of degree $\leqq 2 m-1$. Let $S_{m}$, be the set of pairs of integers corresponding to such a set of monomials. Then the only restriction on $S_{m}$, is that the coefficient matrix $X\left(S_{m}\right)$ be nonsingular. There is always such an $S_{m}$, when the hypothesis of Theorem 1 is satisfied.

Certain information about the zeros of a polynomial in one variable will be needed. The following theorem may be found in Marden [7].

THEOREM 2. $\operatorname{Let} f(z)=a_{0}+a_{1} z+\cdots+z^{m}=\prod_{i=1}^{p}\left(z-z_{j}\right)^{m}, F(z)=\left(a_{0}+\epsilon_{0}\right)+$ $\left(a_{1}+\epsilon_{1}\right) z+\cdots+z^{m}$ and let $0<r_{k}<\min \left|z_{k}-z_{j}\right|, j=1, \cdots, p, j \neq k$. Then there exists a positive number $\epsilon$ such that if $\left|\epsilon_{i}\right| \leqq \epsilon$ for $i=0, \cdots, m-1$, then $F(z)$ has precisely $m_{j}$ zeros in the circle $c_{k}$ with center at $z_{k}$ and radius $r_{k}$.

Let $P(\zeta, z)$ be a polynomial in $\zeta$ and $z$. Consider $P$ as a polynomial of degree $m$ in $\zeta$ with coefficients which are polynomials in $z$. A function $\zeta(z)$ such that $P(\zeta(z), z) \equiv 0$ is called an algebraic function. Algebraic functions are discussed by Ahlfors [1], Bliss [3], and others. Briefly, the properties of $\zeta(z)$ we need are as follows. $\zeta(z)$ is an $m$-branched function, each branch of which is analytic at all points except a finite number of branch points and poles of finite order. At a branch point, $\zeta(z)$ is continuous, by Theorem 2, unless the coefficient of $\zeta^{m}$ in $P(\zeta, z)$ vanishes, in which case the function has a pole at the branch point. Algebraic functions form a field, hence a rational function of algebraic functions is again an algebraic function. We especially note that the only singularities that algebraic functions can have are branch points which may be poles of finite order, and poles of finite order. 
For a brief discussion of the number of common zeros of two polynomials in two variables, and other algebraic geometry involved in Theorem 1, see Stroud [10].

3. The Main Result. For this section, let $L_{l}$ denote the Legendre polynomial of degree $l$, with leading coefficient one. We then note that for the square whose vertices are $( \pm 1, \pm 1)$, and with unit weight function, the orthogonal polynomials $P^{(k, m-k)}$ are given by

$$
P^{(k, m-k)}(x, y)=L_{k}(x) L_{m-k}(y) .
$$

Let us consider the orthogonal polynomials $L_{3}(y)$ and $L_{3}(x)+\lambda L_{1}(x) L_{2}(y)$, where $\lambda$ is a parameter. One can then solve for the common zeros as functions of $\lambda$, obtaining the points given in Table 1 . One sees that for $\lambda \in(-9 / 5,9 / 4)$, one can apply Theorem 1 , and obtain a cubature formula of precision $2 m-1=5$, using those points. The weights of the formula are also given in Table 1.

TABLE 1

\begin{tabular}{|l|l||l|}
\hline Point & Weight & \\
\hline$(0,0)$ & $\frac{16(4+5 \lambda)}{9(9+5 \lambda)}$ & $a^{2}=\frac{9+5 \lambda}{15}$ \\
$( \pm a, 0)$ & $\frac{40}{9(9+5 \lambda)}$ & $b^{2}=\frac{9-4 \lambda}{15}$ \\
$( \pm b, \pm c)$ & $\frac{25}{9(9-4 \lambda)}$ & $c^{2}=\frac{3}{5}$ \\
$(0, \pm c)$ & $\frac{40(1-\lambda)}{9(9-4 \lambda)}$ & $\lambda \in\left(-\frac{9}{5}, \frac{9}{4}\right)$ \\
\hline
\end{tabular}

For $\lambda=0$, we obtain the cross product Gauss formula, which has positive weights. For $\lambda=1$, we obtain Radon's formula [8]. The 7 points in Radon's formula have positive weights. For $\lambda=-4 / 5$, we obtain an 8-point formula, which so far as the author knows, was previously unknown, and which has positive weights. As $\lambda$ approaches an endpoint of the interval $(-9 / 5,9 / 4)$, two sets of weights become unbounded and common zeros coalesce.

This type of investigation partially motivated the following theorem.

THEOREM 3. Let $R_{2}=[a, b] \times[c, d]$ and $w(x, y)=u(x) v(y)$. Then, for $m \geqq 3$, there exists a cubature formula of precision $2 m-1$ which uses fewer than $m^{2}$ points. Furthermore, the weights are all positive.

Proof. Let $P_{l, 1}(x)$ denote the orthogonal polynomial of degree $l$ over $[a, b]$ with respect to $u(x)$. Let $P_{l, 2}(y)$ denote the orthogonal polynomial of degree $l$ over $[c, d]$ with respect to $v(y)$. We now consider the two orthogonal polynomials over $R_{\mathbf{2}}$ with respect to $w(x, y)$, 


$$
P_{m, 2}(y), \quad P_{m, 1}(x)+\lambda P_{k, 1}(x) P_{m-k, 2}(y),
$$

where $\lambda$ is a parameter, $0<k<m$, and $m+k$ is an even integer. The common zeros of the polynomials (4) are of the form $\left(x_{i j}, y_{i}\right), i, j=1, \cdots, m$, where the $y_{i}$ are independent of $\lambda$, and the $x_{i j}$ are continuous functions of $\lambda$, by Theorem 2 .

We first establish that there is an interval $J$ containing the origin such that $\lambda \in J$ implies the $\left(x_{i j}, y_{j}\right)$ are real and distinct points. For $\lambda=0$, the $\left(x_{i j}, y_{i}\right)$ are real and distinct by the properties of orthogonal polynomials in one variable (see Jackson [6], for example). That the $\left(x_{i j}, y_{i}\right)$ are real and distinct in an open neighborhood of the origin follows from continuity of the $x_{i j}$ and the fact that complex $x_{i j}$ appear in conjugate pairs. Thus, as $\lambda$ increases, or decreases, from $\lambda=0$, one encounters a multiple zero before one obtains complex zeros.

We must show that multiple zeros occur for a finite value of $\lambda$, i.e., that $J$ is not the entire real axis. For multiple zeros to occur, the equations in $\lambda$ and $x$,

$$
\begin{aligned}
& P_{m, 1}(x)+\lambda P_{k, 1}(x) P_{m-k, 2}\left(y_{j}\right)=0, \\
& P_{m, 1}^{\prime}(x)+\lambda P_{k, 1}^{\prime}(x) P_{m-k, 2}\left(y_{i}\right)=0,
\end{aligned}
$$

must have a solution for one of the $y_{i}$. By the properties of orthogonal polynomials in one variable, $P_{m-k, 2}\left(y_{i}\right) \neq 0$ for at least one of the $y_{i}$. We also know that no value of $x$ is a zero of both $P_{k, 1}(x)$ and $P_{k, 1}^{\prime}(x)$. Eliminating $\lambda$ from the system (5), we obtain

$$
P_{m, 1}^{\prime}(x) P_{k, 1}(x)-P_{m, 1}(x) P_{k, 1}^{\prime}(x)=0 .
$$

The degree of the equation is $m+k-1$, an odd number. Thus, the polynomial has at least one real root, $x^{*}$. Since one of $P_{k, 1}\left(x^{*}\right)$ or $P_{k, 1}^{\prime}\left(x^{*}\right)$ is nonzero, we can solve for the corresponding value of $\lambda, \lambda^{*}$ which is finite.

In practice, one might want to find the largest interval $J$ containing zero for which the common zeros of (4) are real and distinct. We need only be concerned here that such an interval exists, and that it is not the entire real line.

Let $J$ denote the largest interval (it is clearly open) such that $\lambda \in J$ implies the polynomials (4) have real and distinct common zeros. We note that, for any finite value of $\lambda$, the common zeros are finite. By Theorem 1, there is a cubature formula of precision $2 m-1$ which uses the points $\left(x_{i j}, y_{i}\right)$ as evaluation points.

We now prove a lemma which will allow us to explicitly state the form of the weights in the formula as functions of the $x_{i j}$, and thus as functions of $\lambda$.

LeMmA 4. Let $S_{m^{2}}=\{(\alpha, \beta): 0 \leqq \alpha, \beta<m\}$ and let $\left(x_{i j}, y_{i}\right), i, j=1, \cdots, m$, be the common zeros of (4), corresponding to $\lambda \in J$. Then $X\left(S_{m^{2}}\right)$ is nonsingular.

Proof. Suppose there exists a $\lambda \in J$ such that $X\left(S_{m^{2}}\right)$ is singular. This is equivalent to the existence of a nontrivial polynomial $Q_{2 m-2}(x, y)=\sum_{(\alpha, \beta) \epsilon S_{m^{2}}} a_{(\alpha, \beta)} x^{\alpha} y^{\beta}$ which vanishes at every point $\left(x_{i}, y_{i}\right)$. Thus, for every $j, Q_{2 m-2}\left(x, y_{i}\right)$ is zero at $x_{i j}$, $i=1, \cdots, m$. Since those $x_{i j}$ are distinct and $Q_{2 m-2}\left(x, y_{i}\right)$ has degree $\leqq m-1$ in $x$, we have $Q_{2 m-2}\left(x, y_{i}\right) \equiv 0$ for $j=1, \cdots, m$. But the coefficient of each power of $x$ in $Q_{2 m-2}$ has degree $\leqq m-1$ in $y$. Thus $Q_{2 m-2}(x, y) \equiv 0$, a contradiction.

On the basis of Lemma 4 , we may solve the weights, $A_{i j}(\lambda)$, by making the approximation exact for monomials corresponding to elements of $S_{m^{2}}$. Thus, we are spared any difficulties which could arise from having to change that set in the interval $J$.

We note that each $A_{i j}(\lambda)$ can be expressed as a rational function of the $x_{i j}(\lambda)$. Since each $x_{i j}$ is an algebraic function of $\lambda$, so is $A_{i j}$. 
The proof now breaks into two cases: case (i) for some $\lambda \in J$, one or more of the $A_{i j}(\lambda)=0$; case (ii) for all $\lambda \in J, A_{i j}(\lambda) \neq 0, i, j=1, \cdots, m$. Assume case (i) is encountered. Let $\lambda^{*} \in J$ be the smallest number in absolute value such that one or more $A_{i j}\left(\lambda^{*}\right)=0$. For $\lambda \in\left(-\left|\lambda^{*}\right|,\left|\lambda^{*}\right|\right)$ all $A_{i j}(\lambda)>0$, by the continuity of the $A_{i j}$. Thus $A_{i j}\left(\lambda^{*}\right) \geqq 0$ for all $i, j=1, \cdots, m$ and by choice of $\lambda^{*}$, at least one $A_{i j}\left(\lambda^{*}\right)$ is zero. Thus the resulting cubature formula effectively uses fewer than $m^{2}$ points.

Now assume that case (ii) is encountered. We first note that for $\lambda \in J, A_{i j}>0$, for $i, j=1, \cdots, m$, by continuity. Let $\lambda^{*} \in$ closure $(J), \lambda^{*} \notin J$, where $\lambda^{*}$ is finite. Then for some $j, i$, and $i^{\prime}, x_{i j}\left(\lambda^{*}\right)=x_{i^{\prime} j}\left(\lambda^{*}\right)$. We will show that $\lim _{\lambda \rightarrow \lambda^{*} ; \lambda \in J} A_{i j}(\lambda)$ exists for each $i, j=1, \cdots, m$.

Precision for constants tells us that $\sum_{i, j=1}^{m} A_{i j}(\lambda)=I(1)$, and hence each $A_{i j}(\lambda)$ is bounded for $\lambda \in J$. The only singularities possessed by algebraic functions are poles of finite order. $\lambda=\lambda^{*}$ is a branch point of some of the $x_{i j}(\lambda)$. Since it is a pole of at most finite order, and since each $A_{i j}(\lambda)$ is bounded as $\lambda \rightarrow \lambda^{*}$ through values in $J$, we see that $\lim _{\lambda \rightarrow \lambda * ; \lambda \in J} A_{i j}(\lambda)$ exists. Let $A_{i j}^{*}=\lim _{\lambda \rightarrow \lambda^{*} ; \lambda \in J} A_{i j}(\lambda)$, and let $x_{i j}^{*}=x_{i j}\left(\lambda^{*}\right)$. Then we have

$$
I\left(Q_{2 m-1}\right)=\sum_{i, j=1}^{m} A_{i j}^{*} Q_{2 m-1}\left(x_{i j}^{*}, y_{i}\right)
$$

for all $Q_{2 m-1}$, since (6) is obtained by taking the limit on the right side of

$$
I\left(Q_{2 m-1}\right)=\sum_{i, i=1}^{m} A_{i j}(\lambda) Q_{2 m-1}\left(x_{i j}(\lambda), y_{i}\right),
$$

which holds for all $Q_{2 m-1}$, for all $\lambda \in J$. Since some of the $\left(x_{i j}, y_{i}\right)$ coincide, (6) effectively uses fewer than $m^{2}$ points. Since $A_{i j}(\lambda)>0$ for all $\lambda \in J, A_{i j}^{*} \geqq 0$, for $i, j=1, \cdots, m$.

4. Numerical Examples. The proof of Theorem 3 indicates an algorithm for computing formulas using fewer than $\mathrm{m}^{2}$ points. Starting with the cross product $(\lambda=0)$, one can easily compute the common zeros of the polynomials (4), then the weights, as $\lambda$ varies. For large values of $m$, the method may break down numerically since the matrix $X\left(S_{m^{2}}\right)$ is ill conditioned. One can observe the behavior of the weights to determine whether case (i) or case (ii) applies. This procedure was used in the numerical examples which follow.

In the tables in this paper, the notation $(-1) 0.123 \cdots$ means $0.123 \cdots \times 10^{-1}$. All calculations were done in double precision on the Univac 1108 at the University of Utah. The calculations were verified by checking the error in the approximation for those monomials which were to be approximated exactly. The errors were sufficiently small that the rounded values given in the tables should be accurate to the given number of digits.

Example 1. We consider the square with vertices at $( \pm 1, \pm 1)$ and weight function $w(x, y)=1$. Letting $L_{l}$ denote the Legendre polynomial of degree $l$ with leading coefficient one, we consider the common zeros of

$$
L_{4}(y), \quad L_{4}(x)+\lambda L_{2}(x) L_{2}(y) .
$$

It is found that case (ii) applies, with 


$$
J=\left(-\frac{27}{490}\left(3(30)^{1 / 2}+5\right), \frac{27}{490}\left(3(30)^{1 / 2}-5\right)\right)
$$

Two 14-point formulas of precision $2 m-1=7$ are obtained, corresponding to the endpoints of $J$. The approximate values of the points and weights are given in Table 2. Note that one formula has four points outside of the square.

TABLE 2

\begin{tabular}{|c|c|c|c|}
\hline & $\mathrm{x}$ & y & Weight \\
\hline$\pm(1) 0.10578$ & 4012371275 & $\pm a$ & $(-1) 0.43784 \quad 15208 \quad 72291$ \\
\hline \pm 0.77459 & 6669241483 & $\pm b$ & $0.3623028638 \quad 12526$ \\
\hline \pm 0.46925 & 3522127911 & $\pm a$ & $0.30407 \quad 0693050225$ \\
\hline 0.00000 & 0000000000 & $\pm b$ & $0.57968 \quad 4582100041$ \\
\hline \multicolumn{4}{|c|}{$\lambda=-\frac{27}{490}(3 \sqrt{30}+5)$} \\
\hline \pm 0.77459 & 6669241483 & $\pm a$ & 0.193252691743030 \\
\hline \pm 0.91506 & 0523380880 & $\pm b$ & 0.169049921219002 \\
\hline 0.00000 & 0000000000 & $\pm a$ & 0.309204306788848 \\
\hline \pm 0.39619 & 1039748320 & $\pm b$ & $0.4830952336 \quad 43544$ \\
\hline \multicolumn{4}{|c|}{$\lambda=\frac{27}{490} \quad(3 \sqrt{30}-5)$} \\
\hline \multicolumn{3}{|c|}{$a^{2}=\frac{15+2 \sqrt{30}}{35}}$, & $a \simeq 0.861136311594053$ \\
\hline \multicolumn{4}{|c|}{$\mathrm{b}^{2}=\frac{15-2 \sqrt{30}}{35}$} \\
\hline
\end{tabular}

Example 2. Let $R_{2}$ and $w$ be the same as Example 1. Consider the common zeros of

$$
L_{5}(y), \quad L_{5}(x)+\lambda L_{1}(x) L_{4}(y) .
$$

It is found that case (i) applies, and again two formulas with fewer than $m^{2}=25$ points are obtained which have positive weights. These formulas are given in Table 3. A third formula is given; although it has a negative weight at the origin, it is interesting because it uses fewer points than either of the other two.

We also note that not all formulas obtained in case (i) have positive weights, which emphasizes the procedure used in the proof to assure positive weights.

The formulas have precision 9. 
TABLE 3

\begin{tabular}{|c|c|c|c|}
\hline & $\mathrm{x}$ & $\mathrm{y}$ & Weight \\
\hline+0.84592 & 7799771709 & $\pm a$ & $(-1) 0.70506 \quad 51405 \quad 64012$ \\
\hline+0.62890 & 1636732253 & $\pm a$ & $(-1) 0.72112 \quad 15110 \quad 07611$ \\
\hline+0.95968 & 1421214621 & $+b$ & 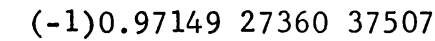 \\
\hline \pm 0.43603 & 0596273468 & $\pm b$ & $0.3685490486 \quad 77049$ \\
\hline+0.77459 & 6669241483 & 0.0 & $0.3160493827 \quad 16049$ \\
\hline 0.00000 & 0000000000 & $\pm a$ & 0.188616439798053 \\
\hline 0.00000 & 0000000000 & $+b$ & $(-1) 0.25860 \quad 69643 \quad 71341$ \\
\hline \multirow[t]{2}{*}{0.00000} & 0000000000 & 0.0 & 0.505679012345679 \\
\hline & \multicolumn{3}{|c|}{$\lambda \cong 0.800000000000000 ; 23$ points } \\
\hline+0.94581 & 3739519925 & $+a$ & $(-1) 0.4992906230 \quad 65150$ \\
\hline+0.46534 & 6624836203 & $\pm a$ & 0.158445182284802 \\
\hline+0.80425 & 3925742002 & $+b$ & $0.18338 \quad 37881 \quad 51247$ \\
\hline+0.68138 & 5892163677 & $\pm b$ & $(-1) 0.88147 \quad 65236 \quad 65422$ \\
\hline+0.96301 & 8409085396 & 0.0 & $0.11445 \quad 63755 \quad 61331$ \\
\hline \pm 0.42861 & 0143223121 & 0.0 & 0.454432513327558 \\
\hline 0.00000 & 0000000000 & $+a$ & $(-1) 0.571052809297435$ \\
\hline \multirow[t]{2}{*}{0.00000} & 0000000000 & $\pm b$ & 0.414194459963155 \\
\hline & \multicolumn{3}{|c|}{$\lambda \cong 0.790123456790123 ; 24$ points } \\
\hline+0.94930 & $73500 \quad 01342$ & $\pm a$ & $(-1) 0.494522019130682$ \\
\hline+0.45817 & 7548931134 & $\pm a$ & $0.1639147318 \quad 81061$ \\
\hline \pm 0.77459 & 6669241483 & $\pm b$ & 0.265904816944092 \\
\hline+0.96777 & 6908976724 & 0.0 & $0.11304 \quad 18390 \quad 46410$ \\
\hline+0.41775 & 4671502987 & 0.0 & $0.4799222296 \quad 00720$ \\
\hline 0.00000 & 0000000000 & $\pm a$ & $(-1) 0.471199025241204$ \\
\hline 0.00000 & 0000000000 & $+b$ & $0.4254477071 \quad 10548$ \\
\hline \multirow[t]{2}{*}{0.00000} & 0000000000 & 0.0 & $-(-1) 0.481503595164821$ \\
\hline & \multicolumn{3}{|c|}{$\lambda \cong 0.870825033167594 ; 21$ points } \\
\hline & $a^{2}=\frac{5}{9}+\frac{2}{63}$ & & $a \simeq 0.906179845938664$ \\
\hline & $b^{2}=\frac{5}{9}-\frac{2}{63}$ & & $b \cong 0.538469310105683$ \\
\hline
\end{tabular}


Example 3. Let $R_{2}=[0, \infty) \times[-1,1]$ and $w(x, y)=e^{-x} /\left(1-y^{2}\right)^{1 / 2}$. This example was chosen to illustrate the theorem for a nontrivial weight function on a nonsymmetric region. Let $\mathfrak{L}_{l}$ denote the Laguerre polynomial of degree $l$ with leading coefficient one, and $T_{l}$ the Chebyshev polynomial of degree $l$ with leading coefficient one. We consider the common zeros of

$$
T_{4}(y), \quad \aleph_{4}(x)+\lambda £_{2}(x) T_{2}(y) .
$$

During the computations investigating the behavior of the weights, it was first thought that case (ii) applied. However, closer numerical investigation revealed that case (i)

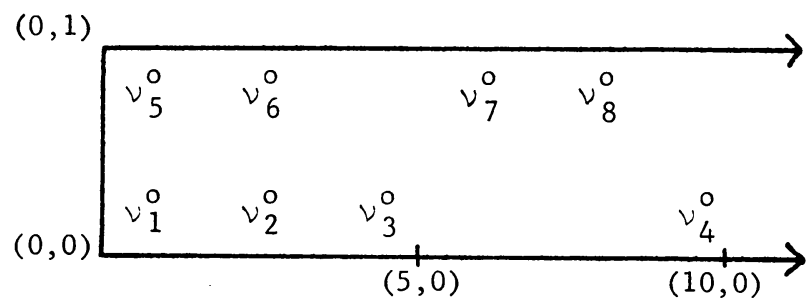

General location of common zeros, $\lambda>0$

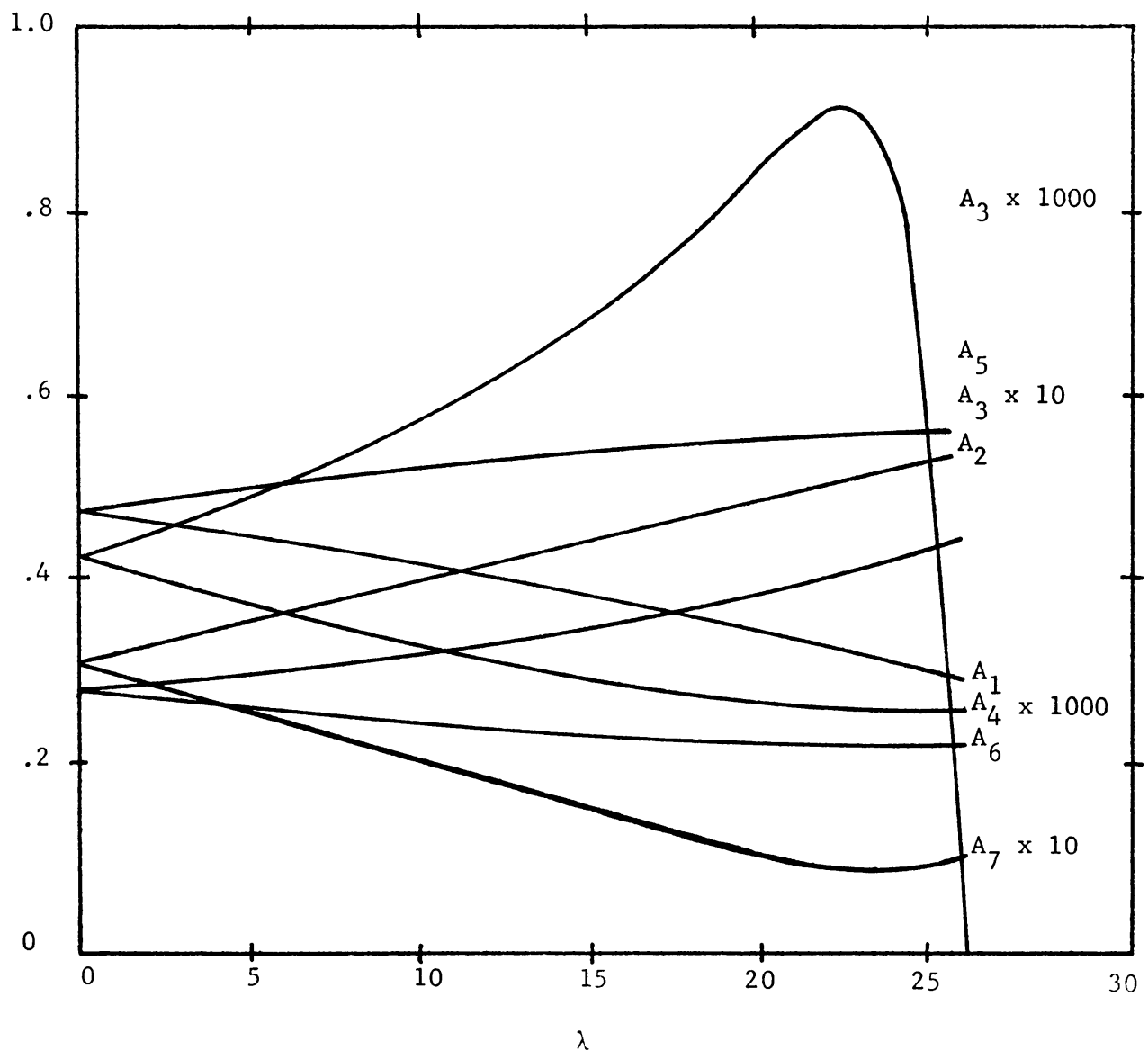

FIGURE 1 
applied. Because of the unexpected behavior of the weights, their values as functions of $\lambda$ are shown in Figure 1. One of the two formulas is given in Table 4, and corresponds to $\lambda^{*}=(2) 0.254558441227157$. The second is obtained by interchanging $a$ and $b$ in the evaluation points, and corresponds to $\lambda=-\lambda^{*}$. The formulas use 14 points and have precision 7.

TABLE 4

\begin{tabular}{|c|c|c|}
\hline $\mathrm{x}$ & $\mathrm{y}$ & Weight \\
\hline 0.113032861459250 & $\pm a$ & $0.2990062097 \quad 72373$ \\
\hline (1) $0.12290 \quad 70412 \quad 16527$ & $\pm a$ & $0.4331001383 \quad 75078$ \\
\hline (1) $0.40847 \quad 5036725518$ & $\pm a$ & $(-1) 0.530442489147755$ \\
\hline (2) $0.10573 \quad 1463591203$ & $\pm a$ & $(-3) 0.24756 \quad 6335222222$ \\
\hline 0.415774556783479 & $\pm b$ & $0.55849 \quad 11440 \quad 03136$ \\
\hline (1)0.22942 8036027904 & $\pm b$ & $0.2187473164 \quad 18902$ \\
\hline (1) 0.628994508293747 & $\pm b$ & $(-2) 0.815970297541073$ \\
\hline \multicolumn{3}{|c|}{$a^{2}=\frac{2-\sqrt{2}}{4}$} \\
\hline \multicolumn{2}{|c|}{$\mathrm{b}^{2}=\frac{2+\sqrt{2}}{4}$} & $\simeq 0.923879532511287$ \\
\hline
\end{tabular}

5. Generalizations. We note that in the proof of Theorem 3, we could just as well have considered the polynomials:

$$
P_{m, 1}(x), \quad P_{m, 2}(y)+\lambda P_{k, 2}(y) P_{m-k, 1}(x),
$$

with the same restrictions on $k$. Which of the two sets of polynomials to be considered in practice might depend on the desire to preserve some symmetry.

Let us consider two situations which might lend themselves to a treatment similar to the proof of Theorem 3.

(S1) Suppose we have under consideration the square $[-a, a] \times[-a, a]$ with weight function $w(x, y)=u(x) u(y)$, where $u(-x)=u(x)$. Since the region and weight function are fully symmetric, it would be desirable to obtain a fully symmetric formula of precision $2 m-1$ which uses fewer than $m^{2}$ points. Accordingly, if $P_{l}$ is orthogonal to all $Q_{l-1}$ on $[-a, a]$ with respect to $u$, we could consider the orthogonal polynomials

$$
P_{m}(x)+\lambda P_{k}(x) P_{m-k}(y), \quad P_{m}(y)+\lambda P_{k}(y) P_{m-k}(x),
$$

where $m+k$ is even. The zeros are fully symmetric, and if they are distinct and finite, the cubature formula obtained by Theorem 1 is also fully symmetric, since it is unique.

(S2) Suppose $R_{2}$ is not a rectangle, and/or $w(x, y)$ is not a product of functions $x$ and $y$, respectively. However, suppose that there are orthogonal polynomials $P_{m, 1}(x, y)$ and $P_{m, 2}(x, y)$ which satisfy the hypothesis of Theorem 1. Further, suppose the 
common zeros are real, and the weights of the associated cubature formula are all positive. One could then consider the polynomials

$$
P_{m, 1}(x, y)+\lambda P_{m, 3}(x, y), \quad P_{m, 2}(x, y)
$$

or the polynomials

$$
P_{m, 1}(x, y)+\lambda P_{m, 3}(x, y), \quad P_{m, 2}(x, y)+\lambda P_{m, 4}(x, y),
$$

where $P_{m, 3}$ and $P_{m, 4}$ may or may not be the same orthogonal polynomial, and neither is equal to $P_{m, 1}$ or $P_{m, 2}$.

The question in both situations is the same. By varying $\lambda$, can one obtain a cubature formula of precision $2 m-1$ which uses fewer than $m^{2}$ points, either through zero weights or coalescence of common zeros? A completely satisfying answer is unknown at this time. We will discuss each situation in turn, the first being the simpler. We note that in either case, complex zeros appear in conjugate pairs, since

$$
\overline{Q_{d}(x, y)}=Q_{d}(\bar{x}, \bar{y}) .
$$

Let $\lambda^{*}$ be a point where the common zeros of (7) are distinct. According to van der Waerden [13], the Jacobian is nonzero at each common zero. Hence, one can apply the Implicit Function Theorem (see Bochner and Martin [4]) and conclude that the common zeros are analytic functions of $\lambda$ in a neighborhood of $\lambda^{*}$.

Assume that the leading coefficients of the $P_{l}$ are all one. Then, a common zero at infinity would have to satisfy the following:

$$
\begin{aligned}
& x^{m}+\lambda x^{k} y^{m-k}=x^{k}\left(x^{m-k}+\lambda y^{m-k}\right)=0, \\
& y^{m}+\lambda y^{k} x^{m-k}=y^{k}\left(y^{m-k}+\lambda x^{m-k}\right)=0,
\end{aligned}
$$

and we see that this would require $\lambda^{2}=1$. Thus, for $\lambda= \pm 1$, the polynomials (7) have common zeros on the line at infinity. Let $J$ denote the largest interval containing the origin such that $\lambda \in J$ implies the polynomials (7) have $m^{2}$ real, distinct, and finite common zeros. Unless case (i) is obtained (zero weights), it would be necessary that $J$ be properly contained in $(-1,1)$. If that were the case, we would also need the common zeros to be continuous functions of $\lambda$ at the point $\lambda^{*}$ where some of the common zeros coalesce. The analogue of Theorem 2 for common zeros of two polynomials in two variables was not found in the literature. Forsythe $[5$, p. 212] proves a partial analogue of Theorem 2, where only the constant coefficients are allowed to vary. It is expected that the full analogue is true, but at the present we must make that assumption. We would also need to know that the limits of the weights as $\lambda \rightarrow \lambda^{*}$, $\lambda \in J$, exist:

In the second instance, one can tell nothing about the behavior of the common zeros of the polynomials (8) or (9) as functions of $\lambda$. Again, they are analytic in the neighborhood of any value of $\lambda$ which yields $m^{2}$ distinct common zeros. Let $J$ be defined as before, and assume continuity at a point where common zeros coalesce. Assume case (i) does not apply or we are finished. If case (ii) applies, we need $J$ to be a proper subset of the real line; we need the common zeros of (8) or (9) to be uniformly bounded in a subinterval $J^{\prime}$ of $J$ having a finite endpoint $\lambda^{*}$, which is also an endpoint of $J$. Again the limits of the weights $\lambda \rightarrow \lambda^{*}, \lambda \in J$, would have to exist to obtain the desired result.

We see that the first situation is contained in the second, although more can be 
determined in the first situation. The above ideas can be incorporated into a theorem which is primarily useful as a guide to computations in search of cubature rules of precision $2 m-1$ using fewer than $m^{2}$ points.

THEOREM 5. Suppose $P_{m, 1}$ and $P_{m, 2}$ satisfy the conditions of Theorem 1 and that $P_{m, 3}$ and $P_{m, 4}$ are orthogonal polynomials, neither of which is the same as $P_{m, 1}$ or $P_{m, 2}$. Further, suppose that the common zeros of $P_{m, 1}$ and $P_{m, 2}$ are all real and that the weights of the associated cubature rule are positive. Let $J$ denote the largest interval containing the origin such that $\lambda \in J$ implies the common zeros of the polynomials (8) or (9) satisfy the hypothesis of Theorem 1 . If the weights of the cubature rule obtained by Theorem 1 are all positive for all $\lambda \in J$, assume the following:

(a) that for $\lambda^{*} \in$ closure $(J), \lambda^{*} \notin J$, the polynomials (8) or (9) have a finite number of common zeros, none of which are at infinity;

(b) the common zeros are continuous functions of $\lambda$ at the point $\lambda=\lambda^{*}$; and

(c) the limits of the weights as $\lambda \rightarrow \lambda^{*}, \lambda \in J$, exist.

Then there exists a cubature formula of precision $2 m-1$ for $R_{2}$ and which effectively uses fewer than $\mathrm{m}^{2}$ points, and which has positive weights.

6. Numerical Examples. The procedure indicated by the theorem was attempted in a number of examples, with success in each instance. Both case (i) and case (ii) were encountered. Two of the examples involved numerical investigation somewhat beyond that indicated by the theorem.

Example 4. Let $R_{2}=[-1,1] \times[-1,1]$ and $w(x, y)=1$. Consider the polynomials

$$
L_{4}(x)+\lambda L_{2}(x) L_{2}(y), \quad L_{4}(y)+\lambda L_{2}(y) L_{2}(x),
$$

where $L_{l}$ is the Legendre polynomial with leading coefficient one. The common zeros, and thus the cubature formulas, are fully symmetric.

For $\lambda \in(-27 / 35,189 / 385)$, the common zeros are distinct and finite. In this range, the weights of the corresponding cubature formulas are always positive. In the limit, as $\lambda \rightarrow(189 / 385)-$, one obtains Tyler's formula [12], given in Table 5. For $\lambda=189 / 385$, the polynomials (10) have multiple zeros on the $x$ and $y$ axes. If one takes the limit as $\lambda \rightarrow(-27 / 35)+$, one obtains a 13-point formula due to a coalescing of 4 zeros into the origin. Four of the points are outside the square, as can be seen in Table 6. The formulas in Tables 5 and 6 have precision 7.

TABLE 5

(Tyler's Formula)

\begin{tabular}{|c|c||c|}
\hline Point & Weight & \multicolumn{2}{c|}{$\mathrm{r}^{2}=\frac{6}{7}$} \\
\hline$(r, 0)_{F S}$ & $\frac{98}{405}$ & $\mathrm{~s}^{2}=\frac{114-3 \sqrt{583}}{287}$ \\
\hline$(s, s)_{F S}$ & $\frac{178981+2769 \sqrt{583}}{472230}$ & $\mathrm{~s}^{2}=\frac{114+3 \sqrt{583}}{287}$ \\
\hline$(t, t)_{F S}$ & $\frac{178981-2769 \sqrt{583}}{472230}$ & $t^{2}=\frac{1}{}$ \\
\hline
\end{tabular}


TABLE 6

\begin{tabular}{|l|c||l|}
\hline Point & Weight & $a^{2}=\frac{3}{2}$ \\
\hline$(0,0)$ & $\frac{392}{405}$ & $b^{2}=\frac{3}{7}\left(1+\sqrt{\frac{10}{31}}\right)$ \\
$(a, a)_{F S}$ & $\frac{16}{2025}$ & $c^{2}=\frac{3}{7}\left(1-\sqrt{\frac{10}{31}}\right)$ \\
\hline$(b, c)_{F S}$ & $\frac{1519}{4050}$ & $c^{2}$ \\
\hline
\end{tabular}

Example 5. Let $R_{2}=[-1,1] \times[-1,1]$ and $w(x, y)=1$. Consider the polynomials (11)

$$
L_{5}(x)+\lambda L_{3}(x) L_{2}(y)+\mu L_{1}(x) L_{4}(y), \quad L_{5}(y)+\lambda L_{3}(y) L_{2}(x)+\mu L_{1}(y) L_{4}(x) .
$$

The formulas obtained are fully symmetric. It was found that case (i) was obtained for a range of values for $\mu$.

For $\mu \cong-0.538806653167134, \lambda=0$, a 21-point formula of precision 9 is obtained by virtue of a set of four weights (say $A_{1}$ ) being zero. We note that this formula has a negative weight at the origin. Now letting $\mu$ decrease, and finding a corresponding $\lambda$ such that $A_{1}=0$, we eventually find that corresponding to $\mu \cong$ $-0.755404543280930, \lambda \cong 0.291652159217893$, the weight at the origin has increased to zero, thus yielding a 20-point formula. The formula is given in Table 7, and was previously given by Rabinowitz and Richter [9], who computed it by a different method.

TABLE 7

(Rabinowitz-Richter Formula)

\begin{tabular}{|c|c|c|}
\hline Point & Weight & \\
\hline $\begin{array}{l}(a, 0)_{F S} \\
(b, 0)_{F S} \\
(c . c)_{F S} \\
(r, s)_{F S}\end{array}$ & 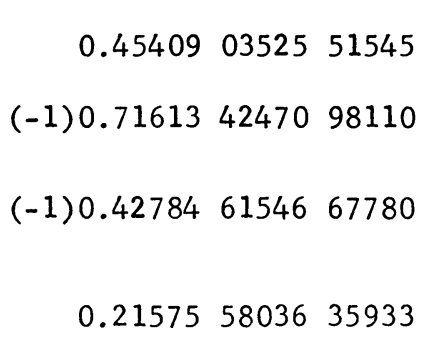 & $\begin{array}{l}a \cong 0.48888 \quad 6342842372 \\
b \cong 0.984539811942252 \\
c \cong 0.939567287421522 \\
r \cong 0.50737 \quad 6773674613 \\
s \cong 0.836710325023989\end{array}$ \\
\hline
\end{tabular}

For $\lambda=0, \mu \cong-0.395061728395062$, a 24-point formula is obtained through the weight at the origin being zero. By decreasing $\mu$ and selecting $\lambda$ to maintain a zero weight at the origin, we expected that the formula of Table 7 would again be obtained. This was not the case. Another 20-point formula of precision 9 was found, corresponding to $\mu \cong-0.723807133586019, \lambda \cong 0.269382462464281$. Note that the values of $\mu$ and $\lambda$ are near the values obtained above, however a different set of 4 
weights are zero, which yields a formula with a different disposition of points than that obtained by Rabinowitz and Richter. The formula is given in Table 8.

TABLE 8

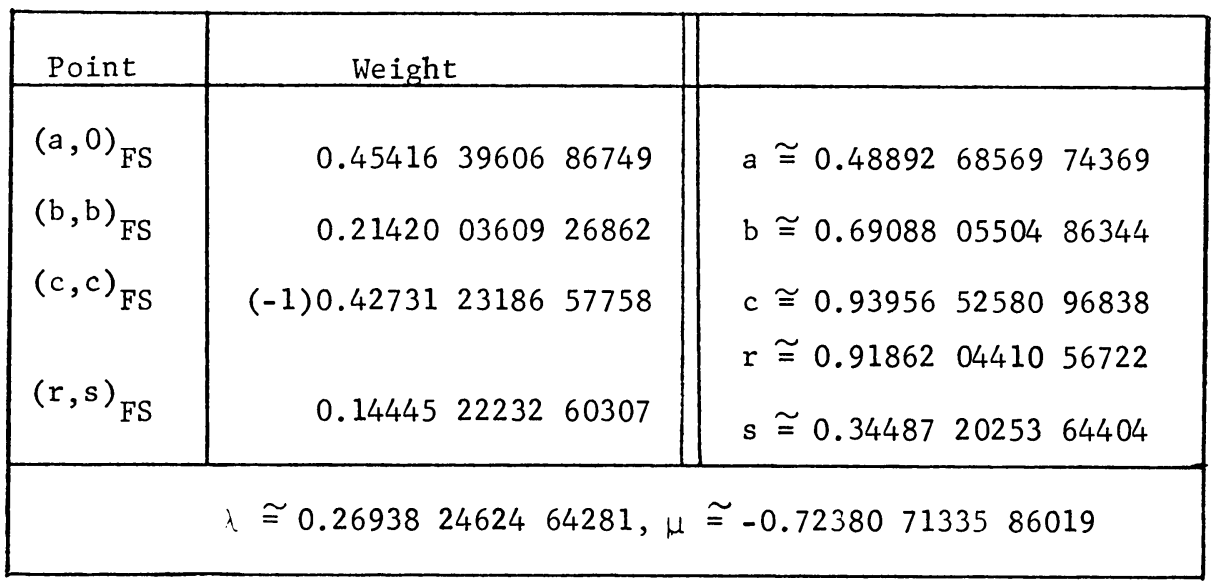

Example 6. Let $R_{2}$ be the triangle with vertices at $(0,0),(1,0)$, and $(0,1)$, with weight function $w(x, y)=1$. The following orthogonal polynomials were given by Appell and Kampé de Feriet [2], and may be found in Stroud [11].

$$
\begin{aligned}
P_{4,1}(x, y)= & 70 x^{4}+140 x^{3} y+90 x^{2} y^{2}+20 x y^{3}+y^{4} \\
& -140 x^{3}-180 x^{2} y-60 x y^{2}-4 y^{3} \\
& +90 x^{2}+60 x y+6 y^{2}-20 x-4 y+1, \\
P_{4,2}(x, y)= & 35 x^{4}+160 x^{3} y+180 x^{2} y^{2}+64 x y^{3}+5 y^{4} \\
& -80 x^{3}-240 x^{2} y-144 x y^{2}-16 y^{3} \\
& +60 x^{2}+96 x y+18 y^{2}-16 x-8 y+1, \\
P_{4,3}(x, y)= & 15 x^{4}+120 x^{3} y+216 x^{2} y^{2}+120 x y^{3}+15 y^{4} \\
& -40 x^{3}-216 x^{2} y-216 x y^{2}-40 y^{3} \\
& +36 x^{2}+108 x y+36 y^{2}-12 x-12 y+1, \\
P_{4,4}(x, y)= & P_{4,2}(y, x), \\
P_{4,5}(x, y)= & P_{4,1}(y, x) .
\end{aligned}
$$

We consider the common zeros of two polynomials of the form

$$
\begin{aligned}
P_{4,6}(x, y) & =\tau P_{4,1}(x, y)+\lambda P_{4,2}(x, y)+\mu P_{4,3}(x, y)+\gamma P_{4,4}(x, y), \\
P_{4,7}(x, y) & =\tau P_{4,5}(x, y)+\lambda P_{4,4}(x, y)+\mu P_{4,3}(x, y)+\gamma P_{4,2}(x, y) \\
& =P_{4,6}(y, x)
\end{aligned}
$$

in an attempt to find a formula of precision 7 which uses fewer than 16 points. The form of the polynomials (12) was chosen to obtain a formula with the same symmetry as the region, i.e., if $(a, b) \in R_{2}$, then $(b, a) \in R_{2}$, and if $(a, b)$ is a common zero of (12), so is $(b, a)$. Including $P_{4,5}(x, y)$ in the first and $P_{4,1}(x, y)$ in the second polynomial leads to no more generality than the above. 
We found that the common zeros of (12) for $\tau=1, \lambda=\mu=\gamma=0$ are all real, distinct, and inside the triangle. However, the cubature rule obtained by using these points has two negative weights. By decreasing $\lambda$, decreasing $\mu$, or increasing $\gamma$, while maintaining $\tau=1$, and the other two of $\lambda, \mu$, and $\gamma$ at zero, it was found that the two negative weights increased, and became zero for some value of each of the three parameters. Thus, three different 14-point rules can be obtained in this manner, each of which has positive weights and one of which has all its points interior to the triangle. The latter formula is given in Table 9. Using the same procedure as in Example 5 , an attempt was made to obtain a formula using fewer than 14 points. One approach which was used, but without success, was to start with the formula given in Table 9, change $\lambda$ slightly, and obtain a new 14-point formula by proper choice of $\mu$. Because this is simply a trial and error method, a complete investigation was impractical. However, it appeared that one of two things would happen: (1) a common zero would tend to infinity; or (2) a common zero would coalesce with the common zero which had a zero weight. Thus, the effort failed to improve on the results indicated by Theorem 5 .

The same procedure was tried, starting with a 14-point formula occurring for $\lambda=-0.08, \mu \cong(-2) 0.715242534751567$. This formula is given in Table 10. While the disposition of the points is similar to that of Table 9, the zero weight occurs for a "different" point, as determined by its position relative to the other points. The points in the formula are interior to the triangle, although the two common zeros with zero weights are outside the triangle. Again no formula with fewer than 14 points was obtained, for the same reasons as in the other attempt.

A very large number of 16-point formulas were computed for various values of $\lambda, \mu$,

TABLE 9

\begin{tabular}{|c|c|c|}
\hline Point & Weight & \\
\hline$\left(a_{1}, a_{1}\right)$ & $(-1) 0.26332 \quad 15013 \quad 60460$ & $a_{1} \cong(-1) 0.646341098016171$ \\
\hline$\left(a_{2}, a_{2}\right)$ & $(-1) 0.666750609902085$ & $0.25047 \quad 87642 \quad 60821$ \\
\hline$\left(a_{3}, a_{3}\right)$ & $(-1) 0.598398472297514$ & $0.40528 \quad 8113134598$ \\
\hline$\left(a_{4}, a_{4}\right)$ & $(-1) 0.302244308027287$ & 0.483428507060240 \\
\hline$\left(b_{1}, c_{1}\right)$ & $(-1) 0.387139102462897$ & $b_{1} \cong(-1) 0.490241549057468$ \\
\hline$\left(c_{1}, b_{1}\right)$ & & $c_{1} \cong 0.312418129002285$ \\
\hline$\left(b_{2}, c_{2}\right)$ & $(-1) 0.22310 \quad 31308 \quad 16147$ & $b_{2} \cong(-1) 0.272654917225016$ \\
\hline$\left(c_{2}, b_{2}\right)$ & & 0.649829918830148 \\
\hline$\left(b_{3}, c_{3}\right)$ & $(-2) 0.93095 \quad 6404694027$ & $b_{3} \cong(-2) 0.748092005042521$ \\
\hline$\left(c_{3}, b_{3}\right)$ & & 0.922929224698637 \\
\hline$\left(b_{4}, c_{4}\right)$ & $(-1) 0.36538 \quad 2927009296$ & 0.166718687651425 \\
\hline$\left(c_{4}, b_{4}\right)$ & & 0.775796880494268 \\
\hline$\left(b_{5}, c_{5}\right)$ & $(-1) 0.515921753448585$ & 0.151969575382297 \\
\hline$\left(c_{5}, b_{5}\right)$ & & $0.5691013418 \quad 00312$ \\
\hline
\end{tabular}


TABLE 10

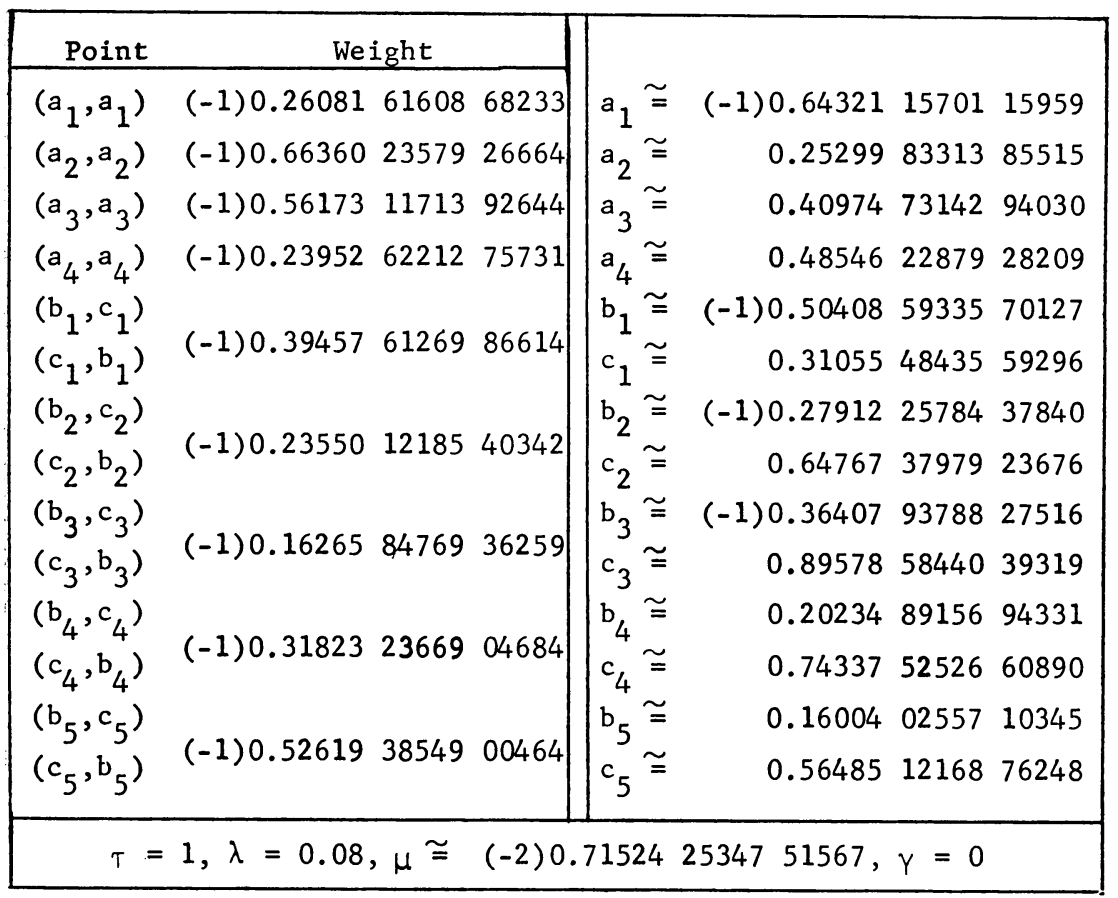

and $\gamma$ in (12) in an effort to determine the behavior of the weights as functions of those parameters. Although the investigation was far from complete, none of the computations gave any indication of the existence of a formula using fewer than 14 points.

Department of Mathematics

Naval Postgraduate School

Monterey, California 93940

1. LARS V. Ahlfors, Complex Analysis: An Introduction to the Theory of Analytic Functions of One Complex Variable, 2nd ed., McGraw-Hill, New York, 1966. MR 32 \# 5844.

2. P. APPELL \& J. KAMPÉ DE FERIET, Fonctions Hypergéométriques et HypersphèriquesPolynomes d'Hermite, Gauthier-Villars, Paris, 1926.

3. Gilbert Ames Bliss, Algebraic Functions, Amer. Math. Soc. Colloq. Publ., vol. 16, Amer. Math. Soc., Providence, R.I., 1933.

4. Salomon Bochner \& William Ted Martin, Several Complex Variables, Princeton Math. Series, vol. 10, Princeton Univ. Press, Princeton, N.J., 1948. MR 10, 366.

5. A. R. Forsythe, Theory of Functions of Two Complex Variables, Cambridge Univ. Press, Cambridge, 1914.

6. Dunham Jackson, Fourier Series and Orthogonal Polynomials, Carus Monograph Series, no. 6, Math. Assoc. of Amer., Buffalo, New York, 1941. MR 3, 230.

7. Morris Marden, Geometry of Polynomials, 2nd ed., Math. Surveys, no. 3, Amer. Math. Soc., Providence, R.I., 1966. MR 37 \#1562.

8. J. Radon, "Zur mechanischen Kubatur," Monatsh. Math., v. 52, 1948, pp. 286-300. MR 11, 405 .

9. Philip Rabinowitz \& NiRa Richter, "Perfectly symmetric two-dimensional integration formulas with minimal numbers of points," Math. Comp., v. 23, 1969, pp. 765-779. MR 41 \#2928.

10. A. H. STRoud, "Integration formulas and orthogonal polynomials for two variables," SIAM J. Numer. Anal., v. 6, 1969, pp. 222-229. MR 41 \#6400.

11. A. H. STRoUd, Approximate Calculation of Multiple Integrals. (Manuscript.)

12. G. W. Tyler, "Numerical integration of functions of several variables,"Canad. J. Math., v. 5, 1953, pp. 393-412. MR 15, 67.

13. B. L. VAN DER WAERDEN, Moderne Algebra. Vol. 2, Springer, Berlin, 1931; English transl., Ungar, New York, 1950. 